better to form one large opening by removing the portion lying between the openings, and transplant as before.

The method of transplantation here suggested is based upon an experimental study in eighteen animals. In all eighteen cases the transplantation was successful.-..Gas. des hopitaux, 1892 , No. 1 $3^{8}$.

\title{
IV. Tuberculous Strictures of the Bowels and Their
}

Treatment. By Dr. F. Konic. K. observed and operated upon five cases of tuberculous stricture of the bowel, a disease, the clinical appearances of which are so typical as to present a picture with striking characteristics. The patients' ages varied from twenty to forty years, only exceeding this in one case. In this a woman of fifty-two ycars. The patient had suffered from gastric symptoms which developed slowly; pallor and emaciation appearing simultaneously. Later there occurred attacks of colicky pains pointing without doubt to stricture of the bowel. With varying frequency, several times a day and again less often, the abdomen became the site of painful distention; loops of bowel with serpentine movements and a splashing noise is noticed upon succussion. The attack tcrminates by the contents of the bowel being forced through the stricture; in the meantime a characteristic noise as if a fluid is pressed out of a syringc becomes audible upon auscultation. Immediately the abdomen flattens and the patient is relicved for a time. Operation discloses conditions corresponding to the picture of the disease. The stricture of the bowel originating from the tuberculous ulcer of the bowel is found with considerable lessening of the lumen from cicatrization. Above this point the bowel is greatly dilated with hypertrophy of the muscular coat; below, the bowel is contracted or rather atrophied. Typical circular resection of bowel for the removal of the olstruction is indicated as well as removal of the affccted mesenteric glands. This procedure is justified by the fact that, as a rule, the tuberculous affection of the bowel in these cases is circumscribed and localized and, as shown by the cicatrization, has an intrinsic tendency to recovery. The diagnosis of a stricture due to tuberculosis will sometimes be suggested by other existing tuberculous affections. Two out of K's five patients died soon after the operation; one from asthenia and one 
from peritonitis due to failure of the suture of the bowel; one of three patients who recovered was in good health two years afterwards; the two others were operated upon more recently. These, likewise, had gained very much in general health and weight.-Dcutsche Zeitschrift f. Chirg., Bd. XXXIV., p. 65 .

V. Primary Sarcoma of the Small Intestine. By Prof. Dr. Madelung (Rostock). Sarcoma attacking primarily the walls of the stomach or intestine is comparatively a very rare disease. This is particularly true of primary sarcoma of the small intestine, heretofore scarcely mentioned in the literature of malignant disease. The development of the tumor in this connection is peculiar, and this, together with the appearances presented, differ greatly from those pre. sented by other neoplasms of the bowel. The author, on the basis of three cases occurring under his own observation, and of eleven cases collected from various sources, presents the following sharp characterization of the disease :

Sarcoma of the small intestine belongs, in most instances, to the round-cell variety, with small cells, and rarely to the spindle-eell type of the disease. They, in all probability have their origin in the sub-mucous layer, and spread in this by preference. The muscular structure is the next to be invaded, and later the mucous membrane; the peritoneum is very rarely attacked, even in advanced cases. As a result of this peculiarity of the method of invasion of the muscular structure of the bowel, the latter becomes paralyzed, and the diseased portion is dilated so as to remind one of an aneurism. Narrowing of the lumen does not occur, even if the mucous membrane becomes extensively diseased. In consequence of the dilatation excessively large tumors are formed early.

Should the tumor force its way through the serous covering, large and irregular intra-peritoneal abscesses with fecal matter as a portion of their contents arise. Metastatic formations in the lymphatic glands of the omentum and mesentery, as well as in the liver and kidneys, occur early. In one instance the small intestine was attacked in two separate places. 